\title{
La calidad de los servicios de traducción e interpretación médico-sanitaria en el contexto del turismo de salud en el área de la Comunidad Valenciana
}

Breve revisión de la situación actual y revisión bibliográfica 


\section{Resumen}

La sociedad contemporánea en España está marcada por la globalización y el multilingüismo. La Comunidad Valenciana en concreto es una entidad autonómica con características poblacionales marcadas por el turismo y la inmigración. En su territorio coexisten distintos grupos demográficos que se correlacionan con distintos perfiles de usuarios de servicios médicosanitarios, incluidos los usuarios del fenómeno demográfico conocido como «turismo de salud».

Ninguno de los documentos que constituyen el marco legislativo de la Unión Europea mencionan explícitamente o contemplan concretamente la provisión de ayuda lingüística para los ciudadanos o residentes legales que buscan asistencia sanitaria en los Estados miembro en los que no tienen acceso a la información debido al desconocimiento del idioma. A falta de unas pautas legislativas claras por parte de la Unión Europea, la legislación pertinente de cada uno de los Estados miembro varía considerablemente y en la mayoría de los casos no hay ninguna legislación al respecto que garantice un servicio igualitario e integral.

La falta de profesionalización del colectivo de traductores e intérpretes conlleva la contratación de intérpretes no profesionales y favorece la proliferación del intrusismo laboral. De este modo, el personal sanitario plurilingüe, los miembros de familia del paciente, incluyendo menores, los voluntarios con criterios de selección mínimos e intérpretes in situ no profesionales y no acreditados asumen el papel de intérpretes ad hoc y se perciben como una solución rápida y barata a pesar de que estas personas carecen de formación, las competencias y las habilidades necesarias.

Tanto los profesionales de la salud como los pacientes y sus familiares ignoran la trascendencia de la labor del intérprete y el hecho de que una interpretación deficiente puede acarrear graves consecuencias para la salud del paciente y la reputación del médico. Así, se vulnera el derecho fundamental del paciente a una asistencia médica de calidad, pues no se asegura su derecho a recibir información completa sobre su estado de salud.

Palabras clave: interpretación médico-sanitaria, turismo de salud, mediación intercultural, inmigración, legislación, normas de calidad, medicina, interpretación profesional, interpretación ad hoc, intrusismo profesional. 
El tema de la calidad de la interpretación en el ámbito médicosanitario es un tema crucial en una sociedad marcada por la globalización y el multilingüismo. He decidido realizar un estudio sobre la importancia de la calidad de los servicios de traducción e interpretación en el ámbito sanitario centrándome en el sector privado, puesto que, durante mi experiencia laboral como intérprete médico-sanitario en un centro privado, he podido detectar serios problemas atribuibles a la falta de servicios de interpretación médico-sanitaria de calidad.

Dado que he desempeñado las funciones de intérprete en uno de los hospitales privados de la Costa Blanca y también he realizado múltiples interpretaciones para mi familia desde que emigramos a España en 2004, puedo observar el mismo problema desde diferentes perspectivas: como intérprete ad hoc para mis familiares en el ámbito de la sanidad pública y como intérprete médico profesional en el ámbito de la sanidad privada. Los clientes en ambos casos presentan perfiles totalmente diferentes, pero unidos por la misma necesidad: un servicio médico de calidad con mediación lingüística. Además, cabe destacar que la interpretación médica en el ámbito de medicina privada (dentro del contexto del turismo de salud) es un tema sobre el que existen pocos trabajos de investigación y, por tanto, escasa bibliografía.

Con esta modesta aportación pretendo contribuir a paliar, en cierto modo, este vacío. De esta manera, en el presente trabajo se detectan y se analizan los problemas de interpretación médico sanitaria desde el punto de vista del propio intérprete que experimenta e identifica diferentes factores socioprofesionales que, en su opinión, dificultan el reconocimiento de la profesión de intérprete para los servicios sanitarios públicos y privados y del «valor social de la interpretación» (Mikkelson, 1999):

1. Falta de concienciación por parte de los proveedores de servicios médicos sobre la importancia de la calidad de la interpretación en el proceso comunicativo.

2. Falta de concienciación por parte de los pacientes que hablan lenguas minoritarias sobre los riesgos a los que se exponen cuando su hospital trabaja con intérpretes no profesionales y no acreditados.

3. Falta de concienciación por parte de los servicios sanitarios y de los propios pacientes de la complejidad inherente a la interpretación como acto comunicativo polifacético.

4. Dificultad para diferenciar los conceptos de traductor e intérprete. Se considera que la interpretación, en el marco de la relación médico-paciente, incluye la 
reformulación oral con función aclarativa mediante los

mecanismos de paráfrasis, desterminologización o recontextualización del mensaje. En numerosas ocasiones se espera del intérprete que este último reestructure y transforme el mensaje para adaptarlo a la situación comunicativa médico-paciente, donde el receptor (el paciente) no tiene el mismo conocimiento que el emisor (el médico). Así, se espera que el intérprete transforme los latinismos y los tecnicismos en homólogos populares, adapte el registro para mejorar la comprensión y de esta manera posibilite el diálogo entre ambos participantes. Pero es importante reflexionar sobre los límites éticos y deontológicos de la labor del intérprete en el ámbito médico-sanitario en concreto: ¿es responsabilidad del intérprete optar o no optar por utilizar unos $u$ otros mecanismos de reformulación o paráfrasis interlingüísticos? ¿Podría la utilización de la desterminologización, la paráfrasis, la reformulación alterar el mensaje original y su finalidad? ¿Cómo se aplicaría el concepto de la fidelidad a la praxis del intérprete médico-sanitario dentro del contexto de la constante necesidad de desterminologización y aclaración para facilitar la comunicación? Por otro lado, las traducciones en el ámbito médicosanitario pueden conservar la finalidad, la situación comunicativa y el registro, pero también pueden ser heterofuncionales.

5. Percepción errónea de las funciones del traductor e intérprete médico-sanitario, que provoca la falta de reconocimiento de la importancia y la necesidad de la figura del traductor e intérprete en esta área.

6. No se puede hablar de cualificación, profesionalidad o calidad si las personas que acceden libremente a la profesión carecen de la formación, la capacidad y la especialización necesarias para desempeñar las funciones del traductor o intérprete médico-sanitario y no se les exige que lo demuestren mediante documentación acreditativa. Tampoco existen criterios de verificación (ya sea de conocimientos lingüísticos reales o de la formación académica en el campo) y selección del personal al puesto de intérprete de plantilla en una clínica u hospital privado.

7. Como resultado de los puntos anteriormente expuestos, en numerosas ocasiones se vulnera el derecho fundamental del paciente a una asistencia médica de calidad, pues no se asegura su derecho a recibir 
información completa y adecuada sobre su estado de salud, sobre su pronóstico y sobre las pruebas e intervenciones a las que podría necesitar someterse.

Los problemas anteriormente desglosados persisten debido a la falta de conocimiento sobre la figura y la función del intérprete y acaban dando lugar a consideraciones erróneas que podrían conllevar graves consecuencias para los pacientes:

a. Las falsas ideas sobre el bilingüismo o pseudobilingüismo llevan a pensar que cualquier persona bilingüe puede desempeñar la labor del intérprete.

b. La idea errónea de que las personas sin formación ni acreditación en las disciplinas de traducción e interpretación puedan acceder a la profesión simplemente porque afirmen que dominan varias lenguas. Esto lleva a los empleadores a no solicitar credenciales de formación especializada ni tampoco de los estudios relacionados, y a no realizar ningún tipo de control de calidad previo a la contratación.

c. La conducta irresponsable por parte del personal médico al afirmar que pueden "apañarse» en inglés, cuando la realidad es que, en la mayor parte de los casos, no dominan esta lengua o les cuesta comunicarse.

d. La falacia del inglés como lingua franca en la que todos se pueden comunicar sin necesidad del intérprete.

e. La no exigencia del cumplimiento estricto de la legislación respecto al derecho del paciente a la información.

f. La falta de profesionalización del colectivo de traductores e intérpretes.

g. La relevancia del presente trabajo consiste en aportar un análisis integral de la situación basado en distintos enfoques que permiten estudiar la realidad desde diferentes perspectivas. Se trata, por tanto, de un estudio que parte de la praxis y de premisas reales y no conjeturas teóricas.

\section{Objetivos}

Los objetivos del presente trabajo de investigación incluyen:

1. Conseguir una mejor comprensión de los servicios de traducción e interpretación y diferenciar entre el ámbito público y el ámbito privado en España.

2. Alcanzar una mejor comprensión de los orígenes del problema de falta de servicios de interpretación sanitaria de calidad (o provisión de servicios deficientes) que no se 
adecúan a los estándares internacionales, tanto en España como en otros países.

3. Contribuir a paliar el vacío en la investigación sobre la interpretación en el ámbito sanitario privado.

4. Reflexionar acerca de la necesidad y la importancia de la calidad de interpretación.

5. Reflexionar sobre las diferentes percepciones del concepto de formación en la traducción e interpretación en distintos países.

6. Revisar las normas de calidad, la legislación vigente y los códigos deontológicos relativos a la provisión de servicios de interpretación médica y a la calidad y formación de los intérpretes.

7. Como objetivo más concreto, se pretende realizar un planteamiento descriptivo inicial del problema apoyado en datos demográficos y normativa aplicable sobre emigración y turismo de salud y realizar una revisión de la bibliografía existente.

\section{Material y método}

La idea de realizar este estudio surgió a partir de mi experiencia laboral como traductora e intérprete médico-sanitaria de un hospital privado. Los problemas prácticos a los que me he enfrentado en mi práctica profesional me han impulsado a realizar una investigación científica. Con el fin de alcanzar los objetivos marcados por dicha investigación, se combinaron técnicas de investigación cualitativa y cuantitativa. Según Hale y Napier (2013: 30-33), la metodología cuantitativa requiere el planteamiento de preguntas muy específicas para confirmar o desmentir una hipótesis. Los datos obtenidos mediante un método de investigación cuantitativo presentan una realidad objetiva. En el caso del método cuantitativo, es importante que el investigador haya desarrollado una hipótesis muy concisa y clara.

Por otra parte, la metodología cualitativa representa una realidad que surge como fruto de construcciones sociales (socially contructed reality) (Berger y Luckman, 1968). Se trata de un enfoque inductivo en el que los datos obtenidos mediante un estudio de investigación pueden generar una teoría. El término "hipótesis» en este enfoque se puede utilizar para referirse a una especulación o conjetura sobre los posibles resultados. La metodología cualitativa requiere la realización de entrevistas con preguntas generales y respuestas complejas que proporcionan una descripción del problema. Los datos obtenidos generan una teoría fundamentada empíricamente. 
De esta forma, se elaboraron las siguientes hipótesis a partir de la experiencia y las observaciones de la realidad laboral predeterminada por el actual constructo social:

1. En las instituciones de la sanidad pública y privada en España, los derechos de los pacientes a una asistencia médica de calidad se ven frecuentemente vulnerados debido a la falta de servicios de traducción e interpretación de calidad.

2. Este hecho podría atribuirse a la falta de concienciación sobre la importancia de la calidad en la traducción e interpretación médico-sanitaria por parte del personal sanitario y la Administración. Esta falta de concienciación es debida, principalmente, a una percepción errónea de la figura y de las funciones que debe desempeñar el intérprete médico-sanitario y la formación, competencias y acreditación que precisa para ello.

3. El desconocimiento del rol y de las funciones que desempeña el intérprete en la comunicación interlingüística tiene como consecuencia la contratación de personas sin ningún tipo de formación previa en traducción e interpretación médica, que no han conceptualizado el término «calidad», por lo que generan un producto deficiente según los criterios de los estándares internacionales (International Organization for Standardization, 2014: 7-9).

4. Estos problemas están íntimamente ligados a la falta de legislación clara al respecto $y$, sobre todo, a la falta de mecanismos legales para exigir su cumplimiento.

De esta manera, la técnica de investigación predominante para corroborar o descartar las hipótesis anteriormente expuestas es la investigación de campo que comprende análisis de realidad social detectando los problemas dentro del contexto de interpretación médico sanitaria en el ámbito de la sanidad privada de la Comunidad Valenciana y ofreciendo soluciones de aplicación práctica. También se ha llevado a cabo una exhaustiva investigación documental que consiste en recopilación de información a través de lectura y análisis crítico del correspondiente material bibliográfico. Por otro lado, dado que la interpretación médico-sanitaria es una actividad que comprende la convergencia entre diversas disciplinas, tales como la interpretación propiamente dicha, la medicia y sus distintas ramificaciones, se trata de llevar a cabo una investigación transdisciplinar recopilando datos a través de un diálogo activo con los participantes (pacientes, médicos, personal sanitario) a partir de contextos y situaciones reales. El período de investigación duró dos años. 
Más concretamente, se ha llevado a cabo un análisis de la situación socioprofesional actual de la interpretación médica en España, tanto en el sector público como privado, a partir de la revisión de los datos demográficos sobre emigración y turismo de salud. A continuación se ha revisado la legislación internacional, europea y española, las normas de calidad y los códigos deontológicos internacionales y nacionales aplicables a la interpretación médica. Por último se ha realizado un trabajo extenso de revisión bibliográfica, a partir de los estudios más representativos que se han llevado a cabo hasta el momento.

Para la revisión bibliográfica, se ha seleccionado una serie de obras que abordan los argumentos más significativos y recurrentes sobre la necesidad de los servicios de interpretación y de mediación intercultural de calidad en el ámbito sanitario. Se ha realizado un análisis de cada una de estas obras y en este trabajo se exponen las ideas más relevantes. El conocimiento de la situación y la experiencia adquiridos en mi puesto de trabajo como intérprete médico en un hospital privado de la Comunidad Valenciana determinaron mis criterios de selección de material y constituyeron un punto de partida para seleccionar las obras en función de su correlación con los problemas que me preocupan, y que pretendo seguir investigando.

\section{Resultados}

La observación de la práctica profesional de la traducción e interpretación médica y la revisión de la legislación y la bibliografía ponen de manifiesto que las hipótesis planteadas quedan parcialmente confirmadas. Esta afirmación está basada en el análisis de los datos empíricos obtenidos por diversos autores de trabajos sobre este tema y queda pendiente la confirmación empírica de estos datos para el territorio que pretendo investigar en el futuro: la Comunidad Valenciana.

Por lo que respecta a la primera hipótesis («En la práctica privada de la medicina en España, los derechos de los pacientes a una asistencia médica apropiada se ven vulnerados con mucha frecuencia por la falta de servicios de interpretación de calidad»), todos los trabajos consultados apuntan en esta dirección. En concreto, Angelelli (2004a; 2012; 2014), Martínez-Gómez Gómez (2008), Moya Bataller (2016), Aguilar Solano (2015) y Castillo García, Taibi y Valero (2005), además de todo el apartado 2.3 sobre la legislación que pone de manifiesto la desprotección del paciente frente a un servicio de interpretación deficiente.

La segunda hipótesis («Este hecho podría atribuirse a la falta de concienciación sobre la importancia de la calidad en la traducción e interpretación médico-sanitaria por parte del personal sanitario y la 
Administración. Esta falta de concienciación es debida, principalmente, a una percepción errónea de la figura y de las funciones que debe desempeñar el intérprete médico-sanitario y las cualificaciones que precisa para ello») se ve refrendada por los estudios empíricos realizados por Pellicer Vidal (2016), Wang (2016), Tellechea Sánchez (2005), Schenker et al. (2007) y Gavlovych (2013).

La tercera hipótesis («El desconocimiento del rol y las funciones que desempeña el intérprete en la comunicación interlingüística tiene como consecuencia la contratación de personas sin ningún tipo de formación previa en traducción e interpretación médica, que no han conceptualizado el término "calidad", por lo que generan un producto deficiente e inaceptable según los estándares internacionales») queda parcialmente confirmada por los resultados de los estudios de Angelelli (2011, 2012), Pöchhacker y Kadric (1999), Niño Moral (2008), Nápoles et al. (2015), Moreno et al. (2015), Jackson et al. (2011), Guske (2010) y Green et al. (2004).

Por último, la cuarta hipótesis («Estos problemas están íntimamente ligados a la falta de legislación íntegra al respecto y el incumplimiento de las normas internacionales de calidad») se ve refrendada por el estudio empírico llevado a cabo por Angelelli (2014).

\section{Discusión y conclusiones}

Este trabajo muestra que queda mucho por estudiar en este campo y pretendo contribuir a avanzar en esta línea con futuras investigaciones. A modo de breve conclusión, recapitularé los puntos clave del trabajo.

1. Como se ha podido comprobar, en muchos países no existe una delimitación clara entre los términos y conceptos intérprete, mediador intercultural y traductor, hecho que genera confusión y desconocimiento generalizado acerca de las funciones de cada una de ellas.

2. A pesar de un elevado número de turistas e inmigrantes en la Comunidad Valenciana, según los datos demográficos consultados, los hospitales públicos no disponen de servicios de interpretación debido a la falta de financiación por parte de la Administración Pública.

3. Muchos facultativos afirman que "se apañan bien en inglés», y otros creen y aseguran que "hablan inglés perfectamente». De esta forma, se comunican con sus pacientes en inglés sin que previamente nadie hubiese determinado o verificado qué nivel lingüístico tienen realmente.

4. Desconocimiento generalizado sobre la figura y las funciones del intérprete y falta de interiorización del concepto de la importancia de la calidad de interpretación en el ámbito 
médico sanitario, que está íntimamente relacionada con la formación, la competencia y la profesionalidad del intérprete.

5. Además, la labor de la interpretación, la mediación intercultural y la traducción (al menos en el ámbito médicosanitario) no están profesionalizadas, hecho que genera libre acceso a la profesión de personas no cualificadas sin ningún tipo de formación específica en traducción e interpretación médico-sanitaria prolifernado de esta manera el instrusismo laboral.

6. Los intérpretes ad hoc (los voluntarios, los miembros de familia incluyendo menores, el personal sanitario $y$ administrativo) no son conscientes de la trascendencia que tienen sus palabras cuando actúan como intérpretes ad hoc.

7. De este modo, los pacientes están desprotegidos frente al peligro de una interpretación deficiente, aunque ellos mismos no se den cuenta. De la misma manera que los médicos no son conscientes del peligro que corre su reputación si el intérprete ad hoc comete un error grave.

8. Todos estos problemas derivan de una legislación deficiente que no prevé los problemas que pueden surgir a causa de una interpretación de calidad insuficiente.

\section{Bibliografía}

Aguilar Solano, Maria. 2015. «Non-Professional Volunteer Interpreting as an Institutionalized Practice in Healthcare: a Study on Interpreters' Personal Narrative». The International Journal for Translation and Interpreting 7 (3): 132-148.

Angelelli, Claudia Viviana. 2004. Medical Interpreting and Crosscultural Communications. Cambridge: Cambridge University Press.

-. 2011. "Can You Ask her about Chronic Illnesses, Diabetes and all that?». En Methods and Strategies of Process Research: Integrative Approaches in Translation Studies, editado por Cecilia Alvstad, Adelina Hild y Elisabet Tiselius, 231-246. Ámsterdam: John Benjamins.

-. 2012. "Challenges in Interpreter's Coordination of the Construction of Pain». En Coordinating Participation in Dialogue Interpreting, editado por Claudio Baraldi y Laura Gavioli, 251268. Ámsterdam: John Benjamins.

-. 2014. "Interpreting in the Healthcare Setting: Access in CrossLinguistic Communication». En The Routledge Handbook of Language and Health Communication, editado por Wen-ying Sylvia Chou y Heidi E. Hamilton, 573-584. Londres: Routledge. 
Berger, Peter, y Thomas Luckman. 1968. La construcción social de la realidad. Buenos Aires: Amorrortu.

Castillo García, Gema Soledad, Mustapha Taibi y Carmen Valero Garcés. 2005. «El papel del intérprete en el ámbito sanitario: reflexiones desde la experiencia». En Traducción como mediación entre lenguas y culturas, editado por Carmen Valero Garcés, 108-113. Alcalá de Henares: Universidad de Alcalá, Servicio de Publicaciones.

Gavlovych, Nina. 2013. La percepción y el rol del intérprete desde el punto de vista ético. Trabajo inédito de fin de grado en Traducción e Interpretación. Castelló de la Plana: Universitat Jaume I.

Green, Judith, Caroline Free, Vanita Bhavnani y Tony Newman. 2004. "Translators and Mediators: Bilingual Young People's Accounts of their Interpreting Work in Health Care». Social Science and Medicine $60 \quad$ (9): 2097-2110. doi:10.1016/j.socscimed.2004.08.067.

Guske, Iris. 2010. "Familial and Institutional Dependence on Bilingual and Bicultural Go Betweens - Effects on Minority Children». mediAzioni 10 (special issue): 325-345. Acceso del 4 de enero de 2017. http://mediazioni.sitlec.unibo.it.

Hale, Sandra B., y Jemina Napier. 2013. Research Methods in Interpreting: A Practical Resource. Londres: Bloomsbury Academic.

Jackson, J. Carey, Diem Nguyen, Nan Hu, Raymond Harris y Genji S. Terasaki. 2011. "Alterations in Medical Interpretation During Routine Primary Care». Journal of General Internal Medicine 26 (3): 259-264. doi: 10.1007/s11606-010-1519-2.

Martínez-Gómez Gómez, Aída. 2008. «Estudio comparativo de la práctica de la interpretación sanitaria en la provincia de Alicante». En Grupo de investigación Frasytram, editado por Pedro J. Mogorron Huerta, 1047-1052. Murcia: Servicio de Publicaciones de la Universidad de Murcia.

Mikkelson, Holly M. 1999. «The Professionalization of Community Interpreting». Journal of Interpretation 6: 119-133.

Moreno, Maria R., Regina Otero-Sabogal y Jeffrey Newman (ed.). 2007. «Assessing Dual-Role Staff-Interpreter Linguistic Competency in an Integrated Healthcare System». Journal of General Internal Medicine 22 (2): 331-335. doi: 10.1007/s11606007-0344-8.

Moya Bataller, Helena. 2016. La situación de la interpretación sanitaria en la ciudad de Gandía. Trabajo de fin de grado en 
Traducción e Interpretación. Acceso del 8 de enero de 2017. http://repositori.uji.es/xmlui/handle/10234/161639.

Nápoles, Anna M., Jasmine Santoyo-Olsson, Leah S. Karliner, Steven E. Gregorich, Eliseo J. Pérez-Stable. 2015. «Inaccurate Language Interpretation and its Clinical Significance in the Medical Encounters of Spanish-speaking Latinos». Medical Care 53 (11): 940-947. Acceso del 5 de diciembre de 2016. https://www.ncbi.nlm.nih.gov/pmc/articles/PMC4610127/pdf/n ihms711239.pdf.

Niño Moral, Dalila. 2008. «Proyecto de estudio de campo sobre las necesidades de mediación lingüística en los hospitales públicos de la provincia de Alicante». En 25 años de lingüística en España: hitos y retos [recurso electrónico], editado por Rafael Monroy Casas y Aquilino Sánchez Pérez, 1063-1069. Murcia: Universidad de Murcia.

Organización Internacional de Estandarización. 2014. International Standard ISO 13611:2014 (E). Interpreting Guidelines for Community Interpreting (Interprétation Lignes directrices pour l'interprétation en milieu social). Ginebra: Organización Internacional de Estandarización.

Pellicer Vidal, Sara. 2016. Memoria de prácticas. Trabajo inédito. Castelló de la Plana: Universitat Jaume I.

Pöchhacker, Franz, y Mira Kadric. 1999. "The Hospital Cleaner as Healthcare Interpreter». The Translator 5 (2): 161-178. Acceso del 3 de diciembre de 2016.

doi: 10.1080/13556509.1999.10799039.

Schenker, Yael, Frances Wang, Sarah Jane Selig, Rita Ng, y Alicia Fernandez. 2007. "The Impact of Language Barriers on Documentation of Informed Consent at a Hospital with On-Site Interpreter Services». Journal of General Internal Medicine 22 (Suppl. 2): 294-299. doi: 10.1007/s11606-007-0359-1.

Tellechea Sánchez, Teresa. 2005. «El intérprete como obstáculo: fortalecimiento y emancipación del usuario para superarlo». En Traducción como Mediación entre lenguas y culturas (Translation as Mediation or How to Bridge Linguistic and Cultural Gaps), editado por Carmen Valero Garcés, 114-121.

Wang, Xixi. 2016. "The Impact of Using Ad Hoc Interpreters and Professional Interpreters on Hospital Costs and Patient Satisfaction Rates of Limited-English-Proficient Patients in the Emergency Department». International Journal of Economics, Commerce and Management IV (3): 245-257. Acceso del 09 de noviembre de 2016.2 http://ijecm.co.uk/wpcontent/uploads/2016/03/4316.pdf. 